\title{
ON GROUPS WITH ALL SUBGROUPS ALMOST SUBNORMAL
}

\section{ELOISA DETOMI}

(Received 1 May 2001; revised 11 April 2003)

Communicated by R. B. Howlett

\begin{abstract}
In this paper we consider groups in which every subgroup has finite index in the $n$th term of its normal closure series, for a fixed integer $n$. We prove that such a group is the extension of a finite normal subgroup by a nilpotent group, whose class is bounded in terms of $n$ only, provided it is either periodic or torsion-free.
\end{abstract}

2000 Mathematics subject classification: primary 20E15, $20 \mathrm{~F} 19$.

A subgroup $H$ of a group $G$ is said to be almost subnormal if it has finite index in some subnormal subgroup of $G$. This occurs when $H$ has finite index in some term $H^{G, n}$, $n \geq 0$, of its normal closure series in $G$; recall that $H^{G, 0}=G$ and $H^{G, n}=H^{H^{G, n-1}}$.

A finite-by-nilpotent group has every subgroup almost subnormal, and for finitely generated groups the converse holds (see $[8,6.3 .3]$ ). Note that, if a group $G$ has a finite normal subgroup $N$ such that $G / N$ is nilpotent of class $n$, then each subgroup $H$ of $G$ has finite index in $H^{G, n}$. For $n=1$, the converse is settled by a well-known theorem of Neumann [10]: a group $G$, in which every subgroup $H$ has finite index in its normal closure $H^{G}$, is finite-by-abelian. Later, Lennox [7] considered the case in which $n$ is larger than 1 and there is also a bound on the indices. He proved that there exists a function $\mu$ such that if $\left|H^{G, n}: H\right| \leq c$ for every subgroup $H$ of a group $G$, where $n$ and $c$ are fixed integer, then the $\mu(n+c)$-th term $\gamma_{\mu(n+c)}(G)$ of the lower central series of $G$ is finite of order at most $c$ !. Recall that a theorem by Roseblade states that a group $G$ in which $H=H^{G, n}$ for every subgroup $H$, is nilpotent and $\gamma_{\rho(n)+1}(G)=1$, for a well-defined function $\rho$. Recently, Casolo and Mainardis in [2,3] gave a description of the structure of groups with all subgroups almost subnormal, proving, in particular, that such groups are finite-by-soluble.

(C) 2004 Australian Mathematical Society $1446-7887 / 04 \$ A 2.00+0.00$ 
In this paper we consider the class $A_{n}, n \geq 1$, of groups $G$ in which $\left|H^{G, n}: H\right|$ is finite for every subgroup $H$ of $G$, but no bound on the indices $\left|H^{G, n}: H\right|$ is assumed. In particular, we give a generalization of Neumann's theorem to periodic $A_{n}$-groups:

THEOREM 1. There exists a function $\delta$ of $n$, such that if $G$ is a torsion group with the property that $\left|H^{G, n}: H\right|<\infty$ for every subgroup $H$ of $G$, then $\gamma_{\delta(n)}(G)$ is finite.

We then consider torsion-free groups. By a result due to Casolo and Mainardis [2], torsion-free $A_{n}$-groups have every subgroup subnormal and so they turn out to be nilpotent, by a recent result by Smith [14] (see also Casolo [1]). Here, we give a different proof of their nilpotency and, in particular, a bound on their nilpotency class, thus generalizing Neumann's theorem to torsion-free $A_{n}$-groups:

THEOREM 2. There exists a function $\eta$ of $n$ such that each torsion-free group $G$ in which $\left|H^{G, n}: H\right|<\infty$ for every subgroup $H$, is nilpotent of class at most $\eta(n)$.

This also gives a different proof of Roseblade's theorem for torsion-free groups with all subgroups subnormal of bounded defect.

Finally, we observe that $S$ mith in [13] gives examples of $A_{2}$-groups which are not finite-by-nilpotent. Thus, Theorem 1 and Theorem 2 are no longer true if we drop the assumptions that $G$ is either periodic or torsion-free. Also, Casolo and Mainardis, in [2], construct a non-hypercentral $A_{2}$-group. On the other hand, in Proposition 13 we shall prove that locally nilpotent $A_{n}$-groups are hypercentral, partially answering the question posed in[8, page 191]. Recall that Heineken-Mohamed groups [6] are example of groups in which every subgroups is almost subnormal but they do not belong to any of the classes $A_{n}$.

\section{1. $A_{n}^{+}$-groups}

In order to achieve our result on periodic $A_{n}$-groups, we find it convenient to study a larger class of groups. We denote by $A_{n}^{+}$the class of all groups $G$ in which there exists a finite subgroup $F$ with the property that every subgroup $H$ containing $F$ has finite index in the $n$th term $H^{G, n}$ of its normal closure series. By abuse of notation, we shall denote the above by $(G, F) \in A_{n}^{+}$. Note that $A_{n} \subseteq A_{n}^{+}$but $A_{n} \neq A_{n}^{+}$. Indeed, the group described in [4, Proposition 4] is a periodic $A_{2}^{+}$-group but it is not finite-by-nilpotent, and so, by Theorem 1 , it does not belong to $A_{n}$.

Also, we denote by $\mathfrak{U}_{n}^{+}$the class of all groups $G$ in which there exists a finite subgroup $F$ such that every subgroup of $G$ containing $F$ is subnormal of defect at most $n$ in $G$. Clearly, $\mathfrak{U}_{n}^{+} \subseteq A_{n}^{+}$, but $\mathfrak{U}_{n}^{+} \neq A_{n}^{+}$, since Smith's groups [13] are locally nilpotent $A_{2}$-groups which are not finite-by-nilpotent while, for $\mathfrak{U}_{n}^{+}$-groups, the following holds: 
THEOREM 3 (Detomi [4]). There exists a function $\beta(n)$ of $n$, such that if $G$ belongs to $\mathfrak{U}_{n}^{+}$and it is either a locally nilpotent group or a torsion group with $\pi(G)$ finite, then $\gamma_{\beta(n)}(G)$ is finite. In particular, if $G$ is locally nilpotent, then $G$ is nilpotent and its nilpotency class is bounded by a function depending on $n$ and $|F|$.

Here $\pi(G)$ denotes the set of primes dividing the orders of the elements of $G$.

The following are two known result which we include without proofs. If $N$ is a subgroup (normal subgroup) with finite index in $G$, then we write $N \leq_{f} G\left(N \unlhd_{f} G\right)$.

LEMMA 4. Let $G$ be a countable residually finite group and let $H$ be a finite subgroup of $G$. Then $H=\bigcap_{N \unlhd_{f} G} H N$.

LEMMA 5. Let $G$ be a group and let $F$ be a finitely generated subgroup of a subgroup $H$ of $G$. If $\left[G,{ }_{n} V\right] \leq V$ for every finitely generated subgroup $V$ of $H$ such that $F \leq V$, then $\left[G,_{n} H\right] \leq H$.

We establish an elementary property of periodic $A_{n}^{+}$-groups:

LEMMA 6. A periodic $A_{n}^{+}$-group is locally finite and finite-by-soluble.

Proof. Let $(G, F) \in A_{n}^{+}$. Then $F \leq_{f} F^{G, n}$ gives that $F^{G, n}$ is finite and that every section $F^{G, i} / F^{G, i+1}$ belongs to $A_{n}$. Since, by the already mentioned result by Casolo-Mainardis, every $A_{n}$-group is finite-by-soluble, the group $G$ has a finite series in which each factor is finite or soluble.

Let $X$ be a finitely generated subgroup of $G$. Clearly $X$ has a finite series with finite or soluble factors. Hence, since a finitely generated torsion soluble group is finite and a subgroup with finite index in a finitely generated group is finitely generated, each factor in this series of $X$ is finite, and so $X$ is finite. This proves that $G$ is locally nilpotent.

Now, since $G$ has a finite series with finite or soluble factors, to prove that $G$ is finite-by-soluble, it is sufficient to show that soluble-by-finite periodic $A_{n}^{+}$-groups are finite-by-soluble.

Let $(G, F) \in A_{n}^{+}$be a torsion group and let $A$ be a soluble normal subgroup with finite index in $G$. We can assume that $A \unlhd G$, since $A_{G}$ has finite index in $G$. Let $\tau$ be a left transversal to $A$ in $G$ and set $H=\langle\tau, F\rangle$. As $H$ has finite index in $K=H^{G, n}, K$ is finitely generated and hence finite, by the local finiteness of $G$. Note that $G=A K$.

We proceed by induction on the defect $d$ of subnormality of $K$ in $G$. If $K$ is normal in $G$, then $G / K \cong A / A \cap K$ is soluble, and we are done. If $d>1$, then, as $K$ has defect of subnormality bounded by $d-1$ in $K^{G}$, we can apply the induction hypothesis to $K^{G}$, obtaining that some term of the derived series of $K^{G}$ is finite (and normal in $G$ ). Therefore, as $G / K^{G} \cong A / A \cap K^{G}$ is soluble, we get that $G$ is finite-by-soluble, which is the desired conclusion. 
With the same argument as in [4, Lemma 9], it is easy to see that:

LEMMA 7. Let $G \in A_{n}^{+}$be a locally finite group. If there exists a subgroup $A$ with finite index in $G$ such that $\gamma_{m+1}(A)$ is finite, then $\gamma_{n m+1}(G)$ is finite.

Roughly speaking, the next proposition says that periodic $A_{n}^{+}$-groups are near to being $\mathfrak{U}_{n}^{+}$-groups.

PROPOSITION 8. Let $G$ be a countable residually finite torsion group and let $G \in$ $A_{n}^{+}$. Then there exists a subgroup $A$ with finite index in $G$ such that $A \in \mathfrak{U}_{n}^{+}$.

ProOF. Assume that the lemma is false and let $G$ be a counterexample. Proceeding recursively we construct

(a) a descending chain $\left\{K_{i} \mid i \in \mathbb{N}\right\}$ of subgroups with finite index in $G$,

(b) an ascending chain $\left\{F_{i} \mid i \in \mathbb{N}\right\}$ of finitely generated subgroups of $\bigcap_{i=0}^{\infty} K_{i}$, and

(c) a sequence of elements $\left\{x_{i} \in\left[K_{i-1, n} F_{i}\right] \backslash K_{i} \mid 1 \leq i \in \mathbb{N}\right\}$.

Set $K_{0}=G$ and let $F_{0}$ be a finite subgroup of $G$ such that $\left|H^{G, n}: H\right|<\infty$ whenever $F_{0} \leq H \leq G$.

Suppose we have already defined $F_{i}, K_{i}$, and $x_{i} \in\left[K_{i-1, n} F_{i}\right] \backslash K_{i}$. As $F_{i}$ is a finitely generated subgroup of $K_{i} \leq_{f} G$, and as $G$ is a counterexample, there exists a subgroup $F_{i} \leq H \leq K_{i}$ which is not subnormal of defect less or equal to $n$ in $K_{i}$, that is, $\left[K_{i, n} H\right] \not L H$. So, by Lemma 5 , there exists a finitely generated subgroup $F_{i+1}$ of $H$ with $F_{i} \leq F_{i+1}$ and $\left[K_{i},{ }_{n} F_{i+1}\right] \not \leq F_{i+1}$. Let us fix an element $x_{i+1} \in\left[K_{i, n} F_{i+1}\right] \backslash F_{i+1}$. Since, by Lemma $6, G$ is locally finite, we can apply Lemma 4 to the finitely generated, hence finite subgroup $F_{i+1}$, and so we get that $x_{i+1} \notin F_{i+1} N$ for a suitable subgroup $N \unlhd_{f} K_{i}$. Then we set $K_{i+1}=F_{i+1} N$, so that $F_{i+1} \leq K_{i+1} \leq_{f} G$ and $x_{i+1} \in\left[K_{i, n} F_{i+1}\right] \backslash K_{i+1}$. Note that $K_{i+1}$ contains all the subgroups $F_{0}, \ldots, F_{i+1}$.

Now we consider the subgroups $K=\bigcap_{i \in \mathbb{N}} K_{i}$ and $H=\left\langle F_{i} \mid i \in \mathbb{N}\right\rangle$. Since $H \geq F_{0}$, by assumption we have that $H$ has finite index in $H^{G, n}$. So, the chain $\left\{H^{G, n} \cap K_{i}\right\}_{i \in \mathbb{N}}$, stretching from $H^{G, n}$ to $H$, is finite and there exists an integer $i$ such that $H^{G, n} \cap K_{i}=H^{G, n} \cap K_{j}$ for every $j \geq i$. But, since $\left[G_{, n} H\right] \leq H^{G, n}$ and $F_{i+1} \leq H \cap K_{i}$, we get that

$$
\begin{aligned}
x_{i+1} \in\left[K_{i, n} F_{i+1}\right] & \leq\left[K_{i, n} H \cap K_{i}\right] \leq\left[G,{ }_{n} H\right] \cap K_{i} \\
& \leq H^{G, n} \cap K_{i}=H^{G, n} \cap K_{i+1},
\end{aligned}
$$

that is $x_{i+1} \in K_{i+1}$, in contradiction to our construction.

THEOREM 9. There exists a function $\delta(n)$ of $n$, such that if $G$ is a periodic $A_{n}^{+}$ group and if either $G$ is locally nilpotent or $\pi(G)$ is finite, then $\gamma_{\delta(n)}(G)$ is finite. In particular, if $G$ is locally nilpotent then $G$ is nilpotent. 
PROOF. Set $\delta(1)=2$ and define recursively $\delta(n)=2 n(\beta(n)-1)+2 \delta(n-1)+1$, where $\beta$ is the function defined in Theorem 3 .

Assume first that $G$ is countable. We shall proceed by induction on $n$. Let $F$ be a finite subgroup of $G$ such that every subgroup $H$ containing $F$ has finite index in $H^{G, n}$.

If $n=1$ then $\left|F^{G}: F\right|<\infty$ and $F^{G}$ is finite. Since $G / F^{G} \in A_{1}$, the quotient $G^{\prime} F^{G} / F^{G}$ is finite by Neumann's theorem. Hence $G^{\prime}=\gamma_{2}(G)$ is finite.

Let now $n>1$ and let $X$ be a finitely generated subgroup of $G$ with $X \geq F$. Because $G$ is locally finite, $X$ is finite. Observe that, for every subgroup $H$ of $X^{G}$ containing $X$, we have $H^{G}=X^{G}$ and so $\left|H^{X^{G}, n-1}: H\right|<\infty$. Thus $X^{G}$ belongs to $A_{n-1}^{+}$and by the inductive hypothesis we get that $\gamma_{\delta(n-1)}\left(X^{G}\right)$ is finite. Now, by a theorem of Hall it follows that $\zeta_{2 \delta(n-1)-2}\left(X^{G}\right)$ has finite index in $X^{G}$. Thus, the index of $C_{G}\left(X^{G} / \zeta_{2 S(n-1)-2}\left(X^{G}\right)\right)$ in $G$ is finite and, denoting by $R=\bigcap_{N \unlhd_{G} G} N$ the finite residual of $G$, we obtain that $\left[R, X^{G}\right] \leq \zeta_{2 \delta(n-1)-2}\left(X^{G}\right)$. In particular,

$$
\left[R, 2 \delta(n-1) X^{G}\right] \leq\left[R, X^{G}, 2 \delta(n-1)-2 X^{G}\right]=1 .
$$

Therefore, if we take $s=2 \delta(n-1)$ elements in $G$, say $x_{1}, \ldots, x_{s}$, and we consider the finitely generated subgroup $X=\left\langle x_{1}, \ldots, x_{s}, F\right\rangle$, then we get $\left[R, x_{1}, \ldots, x_{s}\right] \leq$ $\left[R,{ }_{s} X^{G}\right]=1$, which implies $R \leq \zeta_{s}(G)$.

Now, as $G / R \in A_{n}^{+}$is a countable residually finite torsion group, by Proposition 8 it follows that there exists a subgroup $A$ with finite index in $G$, such that $A / R \in \mathfrak{U}_{n}^{+}$. Also, $A / R$ satisfies the assumptions of Theorem 3 and so $\gamma_{\beta(n)}(A / R)$ is finite. By Lemma 7 it follows that $\gamma_{n(\beta(n)-1)+1}(G / R)$ is finite and then Hall's theorem gives that $\zeta_{2 n(\beta(n)-1)}(G / R)$ has finite index in $G / R$. Therefore, as $R \leq \zeta_{s}(G)$, clearly $\zeta_{2 n(\beta(n)-1)+s}(G)$ has finite index in $G$ and, by a theorem of Baer (see $\left.[12,14.5 .1]\right)$, we conclude that $\gamma_{2 n(\beta(n)-1)+s+1}(G)$ is finite. This proves that $\gamma_{\delta(n)}(G)$ is finite, for every countable group $G$ satisfying the assumption of the theorem.

For the general case, we assume, contrary to our claim, that there exists a group $G$, satisfying the assumption of the theorem, such that $\gamma_{\delta(n)}(G)$ is not finite.

Let $T$ be a countable and not finite subset of $\gamma_{\delta(n)}(G)$. Then we can find a countable set of commutators $x_{i}=\left[y_{1, i}, \ldots, y_{\delta(n), i}\right], i \in \mathbb{N}, y_{j, i} \in G$, such that $T \leq\left\langle x_{i} \mid i \in \mathbb{N}\right\rangle$. Let $Y=\left\langle F, y_{j, i} \mid j=1, \ldots, \delta(n), i \in \mathbb{N}\right\rangle$. As $Y$ is a countable $A_{n}^{+}$-group, by the first part of the proof, $\gamma_{\delta(n)}(Y)$ is finite. Thus $T \subseteq \gamma_{\delta(n)}(Y)$ is finite, against our assumption.

Finally, if $G$ is locally nilpotent, since every finite normal subgroup is contained in some term of the upper central series (by a theorem of Mal'cev and McLain [12, 12.1.6]), it follows that $G$ is nilpotent, and the proof is complete.

As a consequence, we get the announced result on periodic $A_{n}$-groups:

Proof of Theorem 1. Let $G$ be a periodic $A_{n}$-group. By a result of Casolo and 
Mainardis [3], there exists a finite normal subgroup $N$ of $G$ such that $G / N$ has every subgroup subnormal. In particular, $G / N$ is locally nilpotent. Now Theorem 9 gives that $\gamma_{\delta(n)}(G / N)$ is finite and, as $N$ is finite, the result follows.

\section{Torsion-free $A_{n}$-groups}

First we observe some basic properties of isolators in locally nilpotent groups. Recall that the isolator of a subgroup $H$ in a group $G$ is defined to be the set $I_{G}(H)=$ $\left\{x \in G \mid x^{n} \in H\right.$ for some $\left.1 \leq n \in \mathbb{N}\right\}$. If $G$ is a locally nilpotent group then $I_{G}(H)$ is a subgroup of $G$ and if $G$ is also torsion-free then $\gamma_{n}\left(I_{G}(H)\right) \leq I_{G}\left(\gamma_{n}(H)\right.$ ) (see, for example, $[5,9])$.

LEMMA 10. Let $G$ be a locally nilpotent group and let $H \leq G$. Then

(1) if $I_{G}(H)$ is finitely generated, then $\left|I_{G}(H): H\right|<\infty$;

(2) if $G$ is torsion-free and $H$ is cyclic, then $I_{G}(H)$ is locally cyclic.

Proof. (1) As $K=I_{G}(H)$ is a finitely generated nilpotent group, $H$ is subnormal in $K$, say $H=H^{K, n}$ for an integer $n$, and every section $H^{K, i} / H^{K, i+1}$ is finitely generated and nilpotent, for $i=1, \ldots, n-1$. Furthermore, by definition of $I_{G}(H)$, each $H^{K, i} / H^{K, i+1}$ is periodic and hence finite. Thus, $H$ has finite index in $K$.

(2) Let $K$ be a finitely generated subgroup of $I_{G}(H)$. As $H$ is cyclic, we can assume that $H \leq K$. Since $K$ is torsion-free and nilpotent, it has a central series with infinite cyclic factors (see $[12,5.2 .20])$. So, if $K$ is not cyclic, there is a cyclic normal subgroup $N$ of $K$ with infinite index in $K$. Now, since, by (1), $H$ has finite index in $K$, then $H \cap N \neq 1$. Therefore, as $H$ is cyclic, $|K / N| \leq|N H / N|=|H / H \cap N|$ is finite, a contradiction.

We state now a consequence of a well-known argument by Robinson (see [12, 5.2.5]). Recall that the Hirsch length of a polycyclic group $G$ is the number of infinite factors in a series of $G$ with cyclic factors.

LEMMA 11. Let $H$ be a nilpotent group of class $c$. If $H / H^{\prime}$ can be generated by $r$ elements, then the Hirsch length $h$ of $H$ is bounded by a function $g(c, r)$ of $c$ and $r$.

The already mentioned theorem of Mal'cev and McLain [12, 12.1.6] states that each principal factor of a locally nilpotent group is central. The following consequence is well known, but we include the easy proof for the convenience of the reader:

LEMMA 12. Let $G$ be a locally nilpotent group and let $N$ be a finitely generated normal subgroup of $G$. Then there exists an integer $n$ such that $N \leq \zeta_{n}(G)$. Moreover, if $N$ is torsion-free with Hirsch length $h$, then $N \leq \zeta_{h}(G)$. 
PROOF. The theorem of Mal'cev and McLain implies that if $N$ is finite then it is contained in $\zeta_{m}(G)$ for an integer $m$ bounded by the composition length of $N$. Also, when $N$ is torsion-free with Hirsch length $h$, we get that $N / N^{p}$ is finite and so $N / N^{p} \leq \zeta_{h}\left(G / N^{p}\right)$ for every prime $p$; therefore $\left[N,_{h} G\right] \leq \bigcap_{p} N^{p}=1$ by a residual property of torsion-free finitely generated nilpotent groups (see for example [11, page 170]). Since the torsion subgroup of a finitely generated normal subgroup of $G$ is finite, the lemma follows.

\section{PROPOSITION 13. Let $G$ be a locally nilpotent $A_{n}$-group. Then $G$ is hypercentral.}

ProOF. By an already cited result of Casolo and Mainardis, $A_{n}$-groups are finite-bysoluble and so $G$ is soluble. It is sufficient to prove that $G$ has a non trivial centre. We proceed by induction on the derived length of $G$. Let $A$ be the centre of $G^{\prime}$; by inductive assumption, $A \neq 1$. Let $H$ be a finitely generated subgroup of $G$. As $\left|H^{G, n}: H\right|$ is finite, $H^{G, n}$ is finitely generated and so nilpotent; in particular, $\left[A,_{n} H\right]$ is finitely generated. Since $A=\zeta\left(G^{\prime}\right),\left[A,_{n} H\right]^{g}=\left[A,_{n} H^{g}\right] \leq\left[A{ }_{n} H[H,\langle g\rangle]\right]=\left[A{ }_{n} H\right]$ for $g \in G$, and so $\left[A,{ }_{n} H\right]$ is normal in $G$. Thus Proposition 12 gives that $\left[A{ }_{n} H\right] \leq \zeta_{k}(G)$ for some $k \geq 1$. So, if $\left[A,{ }_{n} H\right] \neq 1$, then $\zeta(G) \neq 1$. Otherwise, $\left[A,{ }_{n} H\right]=1$ for any finitely generated subgroup of $G$; thus $A \leq \zeta_{n}(G)$ and we again conclude that $\zeta(G) \neq 1$.

A group $G$ is said $n$-Engel if $[x, n y]=1$ for all $x, y \in G$. We recall that a torsionfree soluble $n$-Engel group $G$ with positive derived length $d$ is nilpotent of class at most $n^{d-1}$ (see $[11,7.36]$ ).

Our interest on Engel groups is motivated by the following fact:

LEMMA 14. A torsion-free $A_{n}$-group is $(n+1)$-Engel.

ProOF. Let $G$ be a torsion-free $A_{n}$-group and let $1 \neq x \in G$. By the definition of the class $A_{n},\langle x\rangle$ has finite index in $\langle x\rangle^{G, n}$, so that $\langle x\rangle^{G, n}$ is a finitely generated subgroup of $I_{G}(\langle x\rangle)$. By the already mentioned result in [2], every subgroup of $G$ is subnormal, so that $G$ is locally nilpotent. Thus, by Lemma $10,\langle x\rangle^{G, n}$ is cyclic, so that $\langle x\rangle \operatorname{char}\langle x\rangle^{G, n}$, and hence $\langle x\rangle$ is subnormal of defect at most $n$ in $G$, that is $\left[G,{ }_{n} x\right] \leq\langle x\rangle$. Therefore, $\left[G,_{n+1} x\right]=\left[G,{ }_{n} x, x\right]=1$, as claimed.

Now we are in a position to prove the announced result on torsion-free $A_{n}$-groups.

PROOF OF THEOREM 2. Let $G \in A_{n}$ be a torsion-free group. As already noted, by a result in [2], $G$ is locally nilpotent.

Note that, if there exists a function $\eta(n)$ such that $\gamma_{\eta(n)+1}(H)=1$, for every finitely generated subgroup $H$ of $G$, then $\gamma_{\eta(n)+1}(G)=1$. Hence, without loss of generality, 
we can assume that $G$ is a finitely generated group. In particular, we get that $G$ is nilpotent and every subgroup of $G$ is finitely generated.

Proceeding by induction on $n$, we prove that there exists a function $\eta(n)$ such that every torsion-free finitely generated $A_{n}$-group has nilpotency class at most $\eta(n)$.

If $n=1$, then Neumann's theorem gives that $G^{\prime}$ is finite. Hence, since $G$ is torsion-free, $G$ is abelian, and so we can set $\eta(1)=1$.

Let now $n>1$ and let $H$ be a subgroup of $G$. Set $H^{G, i}=H_{i}$ for every $i$, so that, by the definition of the class $A_{n}$, we have

$$
H \leq_{f} H_{n} \unlhd H_{n-1} \unlhd \cdots \unlhd H_{2} \unlhd H_{1} \unlhd G .
$$

Note that, for every subgroup $K$ such that $H \leq K \leq H_{1}$, we get $K^{G}=H^{G}=H_{1}$ and $K \leq_{f} K^{K^{c}, n-1}$. Hence $H_{1} / H_{2} \in A_{n-1}$. With the same argument it is easy to see that the factor $H_{i} / H_{i+1}$, for $i=1, \ldots, n-1$, belongs to $A_{n-i}$. By the induction hypothesis, the factor $H_{i} / I_{H_{i}}\left(H_{i+1}\right)$, being a finitely generated torsion-free $A_{n-i}$-group, has nilpotency class at most $\eta(n-i)$; hence,

$$
\gamma_{\eta(n-i)+1}\left(H_{i}\right) \leq I_{H_{i}}\left(H_{i+1}\right) \leq I_{G}\left(H_{i+1}\right) .
$$

Thus,

$$
\gamma_{\eta(n-i)+1}\left(I_{G}\left(H_{i}\right)\right) \leq I_{G}\left(\gamma_{\eta(n-i)+1}\left(H_{i}\right)\right) \leq I_{G}\left(I_{G}\left(H_{i+1}\right)\right)=I_{G}\left(H_{i+1}\right),
$$

for every $i$, so that

$$
\begin{aligned}
\gamma_{\eta(n-1)+1}\left(I_{G}\left(H_{1}\right)\right) & \leq I_{G}\left(H_{2}\right), \\
\gamma_{\eta(n-2)+1}\left(\gamma_{\eta(n-1)+1}\left(I_{G}\left(H_{1}\right)\right)\right) & \leq \gamma_{\eta(n-2)+1}\left(I_{G}\left(H_{2}\right)\right) \leq I_{G}\left(H_{3}\right), \\
\cdots \cdots \cdots \cdots \cdots \cdots \cdots \cdots \cdots & \ldots \cdots \cdots \cdots \cdots \cdots \cdots \\
\gamma_{\eta(1)+1}\left(\gamma_{\eta(2)+1}\left(\cdots\left(\gamma_{\eta(n-1)+1}\left(I_{G}\left(H_{1}\right)\right)\right) \cdots\right)\right) & \leq \gamma_{\eta(1)+1}\left(I_{G}\left(H_{n-1}\right)\right) \\
& \leq I_{G}\left(H_{n}\right)=I_{G}(H),
\end{aligned}
$$

where the last equality is due to the fact that $H \leq_{f} H_{n} \leq I_{G}(H)$.

In particular, for $k=k(n)=\sum_{i=1}^{n-1}(\eta(i)+1)$, the $k$ th term $H_{1}^{(k)}$ of the derived series of $H_{1}$ is a subgroup of $I_{G}(H)$, so that $I_{G}\left(H_{1}^{(k)}\right) \leq I_{G}(H)$. Now, by Lemma 14, $H_{1} / I_{G}\left(H_{1}^{(k)}\right)$ is a soluble torsion-free $(n+1)$-Engel group and so $H_{1} / I_{G}\left(H_{1}^{(k)}\right)$ is nilpotent of class at most $(n+1)^{k-1}$. Thus, for $c=c(n)=(n+1)^{k-1}+1$, we get that $\gamma_{c}\left(H_{1}\right) \leq I_{G}\left(H_{1}^{(k)}\right) \leq I_{G}(H)$. This proves that $\gamma_{c}\left(H^{G}\right) \leq I_{G}(H)$, for every subgroup $H$ of $G$.

Now take $c$ elements of $G$, say $x_{1}, \ldots, x_{c}$, and consider the subgroup $H=$ $\left\langle x_{1}, \ldots, x_{c}\right\rangle$. Clearly we can write $H_{1}=H^{G}$ as a product of the $c$ normal subgroups $\left\langle x_{i}\right\rangle^{G}$. Since $\gamma_{c}\left(\left\langle x_{i}\right\rangle^{G}\right) \leq I_{G}\left(\left\langle x_{i}\right\rangle\right)$ and, by Lemma 10, $I_{G}\left(\left\langle x_{i}\right\rangle\right)$ is a cyclic 
group, then $\left[\gamma_{c}\left(\left\langle x_{i}\right\rangle^{G}\right), x_{i}\right]=1$. Moreover $\left[\gamma_{c}\left(\left\langle x_{i}\right\rangle^{G}\right), x_{i}^{g}\right]=1$ for every $g \in G$. Thus $\gamma_{c}\left(\left\langle x_{i}\right\rangle^{G}\right) \leq \zeta\left(\left\langle x_{i}\right\rangle^{G}\right)$ and $\left\langle x_{i}\right\rangle^{G}$ has nilpotency class at most $c$. Therefore $H_{1}$ is generated by $c$ normal nilpotent subgroups of class at most $c$, and by Fitting's theorem it follows that $H_{1}$ is nilpotent with class $\operatorname{cl}\left(H_{1}\right) \leq c^{2}$.

Now, since $H$ is a $c$-generated torsion-free nilpotent group of $\operatorname{class} \operatorname{cl}(H) \leq$ $\operatorname{cl}\left(H_{1}\right) \leq c^{2}$, Lemma 11 implies that the Hirsch length $h(H)$ of $H$ is bounded by

$$
g_{1}=g\left(c^{2}, c\right)=\frac{c^{c^{2}+1}-1}{c-1} .
$$

Also, by Lemma 10, $\left|I_{G}(H): H\right|<\infty$, so that $h\left(I_{G}(H)\right)=h(H) \leq g_{1}$.

Therefore, $\gamma_{c}\left(H_{1}\right)$ is a finitely generated normal subgroup of $G$ with Hirsch length $h\left(\gamma_{c}\left(H_{1}\right)\right) \leq h\left(I_{G}(H)\right) \leq g_{1}$ and so, by Proposition $12, \gamma_{c}\left(H_{1}\right) \leq \zeta_{g_{1}}(G)$. In particular, $\left[x_{1}, \ldots, x_{c}, y_{1}, \ldots, y_{g_{1}}\right]=1$ for every $y_{1}, \ldots, y_{g_{1}}$ in $G$, so that

$$
\gamma_{c+g_{1}}(G)=1
$$

Finally, since $c=c(n)$ and $g_{1}=g_{1}(n)$ depend only on $n$, the result follows on defining $\eta(n)=c+g_{1}-1$.

\section{Acknowledgement}

This paper presents some of the results of my doctoral dissertation, which was directed by Carlo Casolo. I am indebted to him for his advice and patience during the preparation of my thesis. This work was partially supported by MURST research program 'Teoria dei gruppi e applicazioni'.

\section{References}

[1] C. Casolo, 'Torsion-free groups in which every subgroup is subnormal', Rend. Circ. Mat. Palermo (2) 50 (2001), 321-324.

[2] C. Casolo and M. Mainardis, 'Groups in which every subgroup is $f$-subnormal', J. Group Theory 4 (2001), 341-365.

[3] — , 'Groups with all subgroups $f$-subnormal', in: Topics in infinite groups, Quad. Mat. 8 (Aracne, Rome, 2001) pp. 77-86.

[4] E. Detomi, 'Groups with many subnormal subgroups', J. Algebra 264 (2003), 385-396.

[5] P. Hall, The Edmonton notes on nilpotent groups, Queen Mary College Mathematics Notes (Queen Mary College, London, 1968).

[6] H. Heineken and I. J. Mohamed, 'A group with trivial centre satisfying the normalizer condition', J. Algebra 10 (1968), 368-376.

[7] J. C. Lennox, 'On groups in which every subgroup is almost subnormal', J. London Math. Soc. 15 (1977), 221-231. 
[8] J. C. Lennox and S. E. Stonehewer, Subnormal subgroups of groups (Oxford Univ. Press, Oxford, 1987).

[9] W. Möhres, 'Torsionfreie Gruppen, deren Untergruppen alle subnormal sind', Math. Ann. 284 (1989), 245-249.

[10] B. H. Neumann, 'Groups with finite classes of conjugate subgroups', Math. Z. 63 (1955), 76-96.

[11] D. J. S. Robinson, Finiteness conditions and generalized soluble groups (Springer, Berlin, 1972).

[12] - A course in the theory of groups (Springer, Berlin, 1982).

[13] H. Smith, 'Hypercentral groups with all subgroups subnormal', Bull. London Math. Soc. 15 (1983), 229-234.

[14] —_, 'Torsion-free groups with all subgroups subnormal', Arch. Math. (Basel) 76 (2001), 1-6.

Dipartimento di Matematica

Facoltà di Ingegneria

Università degli Studi di Brescia

via Valotti 9

25133 Brescia

Italia

e-mail: detomi@ing.unibs.it 